\title{
Investigation médicale et juridique de cas de responsabilité civile du médecin*
}

\author{
$1^{\text {re }}$ partie: point de départ et problématique
}

W. E. Ott

Deutsch erschienen

in Nr. 12/2004

1 Olff S. Gesucht wird der Arzt, der einen Fehler zugibt. NZZ am Sonntag, 17 août 2003. p. 57.

2 Löffler W. Die Haftung des Arztes aus ärztlicher Behandlung. 1945.

3 Schilling C. Ärzte geniessen Vertrauen. Tages-Anzeiger, 28 août 2003. p. 7

4 Cf. sur ce point: Geisseler R. Gesamtrevision des Haftpflichtrechts. Revue de l'avocat 2001;11-12:16-21; Müller-Chen M. Brennpunkte der Revision des Haftpflichtrechts. Revue de l'avocat 2001;11-12:10-4; Ott WE. Revision Haftpflichtrecht (et autres indications). Forum HAVE/REAS 2002;3:221-30, avec contribution de plusieurs auteurs et nombreuses indications Jaag T. Staatshaftung nach dem Entwurf für die Revision und Vereinheitlichung des Haftpflichtrechts. Journée des avocats 2003. ZSR 2003;2(1):3ff; Loser-Krogh P. Kritische Überlegungen zur Reform des privaten Haftpflichtrechts - Haftung aus Treu et Glauben, Verursachung und Verjährung. Journée des avocats 2003. Revue du droit suisse $2003 ; 2(2): 127$ ss.

5 Sur ce point: Ott WE. Fehlerhafte Unfallbehandlung. p. $458-9$

* Version écourtée - traduite par la FMH - de l'article paru en allemand dans la revue HAVE Haftung und Versicherung / REAS Responsabilité et assurance, cahier 4, 2003, p. 275-90, publiée ici avec l'aimable autorisation de l'éditeur.

Correspondance:

Me Werner E. Ott, Dr en droit

Badenerstrasse 21

CH-8026 Zurich
Sincèrement: quelle serait votre réaction, en qualité de représentante ou représentant d'une corporation professionnelle reconnue, si l'on vous accusait de violation du devoir de diligence? Sauriez-vous quels sont vos droits et devoirs, quelle procédure suivre? Auriez-vous le courage de reconnaître une faute?

Avouons-le: ces questions ne sont guère agréables et pourtant, les médecins y sont parfois confrontés et doivent pouvoir y donner une réponse professionnelle. Nombre d'entre eux ressentent, aujourd'hui encore, le reproche d'un patient comme étant une attaque personnelle à leur propre intégrité humaine et professionnelle [1]. Cela dit, toutes les professions sont confrontées depuis longtemps à l'évaluation judiciaire de leur activité. W. Löffler [2] a déjà mis en évidence que le médecin n'avait pas de place particulière parmi les justiciables. Le patient «majeur» fait valoir ses droits et est de mieux en mieux informé. L'internet lui offre un nombre incalculable d'informations médicales - de très diverses qualités. Selon une récente étude européenne, 91\% des patients interrogés souhaitent participer aux décisions visant les traitements médicaux et seuls $63 \%$ d'entre eux disent être suffisamment impliqués dans le processus décisionnel [3]. Le présent article est consacré aux questions qui se posent, vues du praticien en droit, en relation avec les éclaircissements médicaux et juridiques de l'expertise extrajudiciaire dans les cas de responsabilité civile du médecin. De nombreux exemples illustreront le propos. La concision rédactionnelle exigée demande à l'auteur de sacrifier à l'exhaustivité, au sens d'un «courage de la lacune». Fondé sur ses propres expériences d'«avocat de patients», son article n'a pas la prétention d'une appréciation universellement valable.

Une première remarque s'impose: le texte ci-après s'en tient au droit en vigueur. L'avantprojet de loi fédérale présenté à l'automne 2000 sur la révision et l'uniformisation du droit de la responsabilité civile contient, il est vrai, plusieurs propositions visant à définir de futures dispositions en matière de procédure et de preuves, à régler la responsabilité de l'organisation et à ins- taurer une responsabilité objective pour les activités particulièrement dangereuses. Mais depuis une consultation terminée à la mi-2001, ces projets aussi ambitieux que controversés ont fini dans les oubliettes du Palais fédéral, sans que l'on en ait jusqu'ici appris grand-chose de plus [4]. Selon l'Office fédéral de la justice (OFJ), le Conseil fédéral devrait sous peu décider de la suite à donner à l'affaire (remarque de la rédaction: il paraît qu'entre-temps, le Conseiller fédéral Blocher aurait remis dans le tiroir le projet actuel).

Deuxième remarque: l'atteinte à la relation de confiance entre médecin et patient en tant que conséquence d'une complication médicale peut facilement conduire à des problèmes de compréhension mutuelle, aspect «psychologique» dont il convient de tenir compte lors de l'étude du cas de sinistre. De plus, médecins et juristes ont les uns et les autres un langage particulier, fait de termes techniques et savants qui risquent de favoriser l'incompréhension. Une constellation encore plus perturbante pour le patient cherchant conseil, généralement profane dans ces deux matières, ne pouvant recourir qu'à son vécu subjectif et se trouvant dès lors, en tant que partie devant fournir des preuves, dans une situation nettement plus difficile que son médecin. En effet, ce dernier dispose d'une avance de connaissances évidemment considérable dans le domaine qui est le sien, étant au courant du dossier médical et de l'ensemble des résultats d'examens. Qu'il en soit conscient s'il se sent attaqué à tort par un patient.

Le but ultime est clair: trouver la vérité, en apprécier adéquatement les éléments médicaux et juridiques, aboutir sur cette base à une solution raisonnable: les violations des règles reconnues de l'art médical classique doivent être indemnisées conformément au droit en matière de responsabilité civile. Seules les complications dues à la fatalité qui, même en application de toute la diligence voulue ne sauraient être évitées, peuvent demeurer sans suites juridiques. Il en va de même de la réalisation d'un risque général, mais la frontière entre l'inévitable et le manque de diligence est parfois floue [5]. 


\section{Investigation médicale}

\section{Démarches et premières vérifications}

Dans la mesure du possible, le patient qui a l'impression d'être la victime d'un traitement erroné ou d'un manquement quelconque de son médecin cherchera tout d'abord le dialogue, en vue de s'informer des circonstances du but thérapeutique non atteint.

Dans nombre de cas, le médecin prendra le temps de se pencher sur les soucis et les questions du patient. Il pourra même peut-être leur trouver une solution. Le patient ira peut-être jusqu'à renoncer à d'autres démarches, si son vis-à-vis manifeste un véritable regret de ce qui s'est passé et que le dommage demeure sans conséquences graves ou permanentes.

Malheureusement, la pratique d'un avocatconseil spécialisé dans ce domaine montre que cette image ne reflète pas toujours la réalité puisque tant du côté du patient que du médecin, la lutte peut être rude, ce qui ne contribue guère, on en conviendra, à une approche constructive des problèmes se posant. Il est dès lors recommandé au patient de demander des conseils avisés suffisamment à temps, auprès d'un autre médecin de confiance, d'une bonne organisation de patients ou d'un avocat spécialisé. Ces derniers seront en mesure de faire les démarches diagnostiques préalables, d'effectuer un premier tri et d'estimer s'il vaut la peine de continuer à élucider le cas ou non.

Ces premières estimations faites, si le patient lésé décide, sur la base de l'appréciation provisoire, de faire examiner plus avant ses éventuelles revendications judiciaires, le cas est alors transmis au médecin ou au répondant légal de l'hôpital concerné, avec demande de l'annoncer à l'assurance responsabilité civile professionnelle ou hospitalière avec le nom, l'adresse et le numéro d'assuré. L'assurance se voit ainsi informée de la situation, des problèmes existants et des premiers résultats de l'enquête médicale et peut en confier l'examen à son propre service médical ou à son médecin-conseil. Pour des raisons de protection des données et de la personnalité, l'accès nécessaire aux données importantes du dossier médical n'est possible qu'avec le consentement du patient. L'assurance demande aussi une prise de position détaillée de son assuré, à savoir des membres du personnel médical concernés. Le patient est avisé de s'armer de patience, l'expérience montrant que cette première phase d'examen du cas prend beaucoup de temps, vu la surcharge chronique des services médicaux. Il est essentiel de savoir que l'annonce du médecin, faite à titre de précaution, ne signifie pas que celui-ci reconnaît une quelconque responsabilité. Elle n'est là que pour créer les conditions d'une investigation correcte. De plus, le médecin s'assure que ses intérêts sont défendus et le coût des démarches couvert par son assurance responsabilité civile, l'assurance ne faisant pas que couvrir les demandes légitimes d'indemnisation et de dommagesintérêts, mais aussi, suivant le contrat établi, la défense contre des demandes en réparation infondées. De leur côté, les assureurs demandent à leurs assurés de ne pas engager des actions de leur propre chef et de ne pas d'emblée reconnaître de responsabilité à l'égard du patient. A l'inverse, l'assurance se réserve le droit, dans ses «conditions générales», de régler un cas évident de responsabilité civile même si le médecin devait s'y opposer.

La première prise de position matérielle de l'assurance décide de la marche à suivre: si les investigations internes confirment la présence d'une responsabilité, elle reconnaîtra celle-ci ou, ce qui arrive fréquemment, elle évoquera une situation non préjudicielle de disponibilité à la négociation, afin de traiter le dommage «en laissant ouverte la question de la responsabilité». Ainsi, les négociations de recours avec les assurances sociales pourront se dérouler toutes options ouvertes et le patient recevra tout de même ses indemnités en dommages-intérêts de manière adéquate. Il faut dire néanmoins que pour le patient, ce scénario optimal n'est pas la règle. L'assurance aura plus souvent tendance, dans un premier temps, à nier la violation des règles de l'art et à n'attribuer le mauvais déroulement de l'événement qu'à des complications malheureuses. S'il arrive qu'une assurance responsabilité civile livre une prise de position médicale et juridique bien documentée et fondée, une autre se contentera d'un simple rejet de la présentation des faits par le patient. La situation est quelque peu différente par rapport au non-respect du devoir d'information du patient, le spécialiste en dommages de l'assurance invoquant volontiers le consentement hypothétique du patient informé, à savoir l'argument selon lequel celui-ci aurait consenti à l'intervention même en étant au courant du risque encouru (et réellement intervenu par la suite) [6]. Dans les cas les plus fréquents - en cet état de la procédure - de rejet total de toute responsabilité, la balle est à nouveau dans le camp du patient qui doit décider s'il veut se lancer dans les dédales d'une expertise indépendante [7] ou s'il préfère renoncer à d'autres démarches. Un deuxième avis indépendant, émanant d’un médecin spécialiste non impliqué, peut lui faciliter la prise de décision. 
La nature des choses veut que l'appréciation du médecin joue un rôle majeur dans l'évaluation d'un cas médical, et la boutade selon laquelle cinq experts émettent cinq avis différents n'est sans doute pas sans une once de vérité. Il est dès lors dans l'intérêt de tous les participants, en cas d'appréciations divergentes, de recourir avant toute chose à une expertise indépendante de haute qualité [8]. L'évaluation de 262 expertises entre 1990 et 1997 a en effet montré que seul un tiers d'entre elles répondaient aux exigences qualitatives, signe qu'il existe des lacunes évidentes en la matière [9].

\section{Expertises privées}

Pour important que soit le travail d'expert en vue d'assurer la qualité de l'activité médicale, il n'en est pas moins fort peu recherché. Accepter un mandat d'expertise signifie toujours du travail supplémentaire, même s'il est convenablement rémunéré. Les exigences personnelles et professionnelles sont élevées, les possibilités de formation jusqu'ici des plus modestes et, dans un petit pays comme le nôtre, il faut savoir parfois composer quant à l'indépendance d'esprit et à la spécialisation des connaissances. De plus, il n'est pas des plus agréables de relever chez un collègue une faute dont on n'est soi-même pas protégé. Les experts les plus reconnus sont dès lors submergés. Certains experts sont considérés comme enclins à défendre le patient d'abord et ne sont pas les bienvenus chez les assureurs, et viceversa. Patient et assurance doivent donc de prime abord convenir de la personne de l'expert, s'entendre sur un catalogue détaillé de questions et la prise en charge des frais. Il n'est pas rare de devoir s'y prendre à plusieurs fois avant de trouver le bon expert qui acceptera effectivement le mandat proposé. Ce procédé est recommandé pour des domaines médicaux exigeant des connaissances particulières, ainsi que dans un contexte de questions très spécifiques, voire les deux. Quiconque préfère aller son propre chemin, sans faire appel à la partie adverse en engageant tout seul une expertise, risque de voir celle-ci demeurer sans suite, puisque qualifiée d'expertise présentée par une seule partie. Expérience faite, ce mode de faire peut diminuer la valeur de preuve de l'expertise en question et ce, tant dans un contexte extrajudiciaire que judiciaire [10].

\section{Expertises de la FMH}

Nombre de ces obstacles tombent si l'on choisit plutôt la voie d'une expertise médicale de la FMH. La Fédération des médecins suisses (FMH) dispose depuis 1982 d'un bureau d'expertises indépendant avec siège à Berne qui a, jusqu'à ce jour, traité

plus de 2700 cas [11]. Le rapport annuel de ce bureau est publié dans le Bulletin des médecins suisses, le dernier en date pour la période 2002 [12]. La marche à suivre est consignée dans un règlement dont la version actuelle date du $1^{\mathrm{er}}$ février 2002 [13]. Il est publié dans le même numéro du Bulletin avec un commentaire [14]. Le Bureau d'expertises de la FMH envoie sur demande une documentation munie d'annexes (adresse pour la Suisse romande: Bureau d'expertises, case postale 64, 1010 Lausanne, tél. 021652 16 74, fax 021652 33 85; pour la Suisse alémanique et le Tessin: FMH-Gutachterstelle, case postale 293, 3000 Berne 16, tél. 03131208 77, fax 03131199 81).

Certes, les expertises de la FMH ont été périodiquement remises en question, surtout de la part des patients, en raison d'une qualité inégale et, dans plus d'un cas, de la longueur de la procédure. Mais ce type d'expertises représente néanmoins, par rapport à une expertise privée, des avantages indéniables. Les étapes de la procédure, clairement définies et normées, sont placées sous la direction du bureau d'expertises neutre de la FMH. La base pour engager la procédure est une demande fondée de la part du patient, qui doit satisfaire au fond et à la forme des normes fixées par le bureau d'expertises. Cette demande doit comporter la confirmation que le médecin ou l'hôpital et son assurance acceptent l'expertise, de manière à éviter le risque d'une expertise lancée par une seule partie. Lorsque le bureau d'expertises entre en matière après un examen préalable sommaire du cas, il confère à la société de discipline médicale concernée le mandat de présenter dans ses rangs un expert idoine ou une équipe d'experts de diverses disciplines. Simultanément, le patient doit s'acquitter des frais de traitement du dossier, qui se montent à 600 francs, TVA en sus, les frais d'experts étant supportés par l'assurance responsabilité civile. Ce règlement permet l'accès au bureau d'expertises à des patients qui n'auraient pas, sinon, les moyens d'engager une procédure pouvant coûter des milliers de francs.

Cela dit, le Bureau d'expertises se réserve le droit de demander une garantie de frais pouvant aller jusqu'à 2000 francs pour des demandes tracassières et sans avenir. Pour les membres de la $\mathrm{FMH}$, l'art. $2 \mathrm{du}$ règlement (obligation pour les membres de la FMH de se soumettre aux procédures d'expertises) précise qu'ils doivent mettre à disposition la totalité des documents et donner le nom de leur compagnie d'assurance responsabilité civile. Il est parfois nécessaire d'insister sur ce point. Le patient est lui aussi soumis à des devoirs de participation. Il met à disposition les documents qu'il a reçus et accepte de se soumettre, à ses frais, à un examen par l'expert. la FMH. Bull Méd Suisses 2002;83(4):134-5.

Cf. l'ouvrage de référence de
Fredenhagen H. Das ärztliche ment. Bull Méd Suisses 2002;83(4):108

14 A propos de la révision du règle- 
15 Ott WE. Voraussetzungen. p. 41.

$163^{\mathrm{e}}$ et $4^{\mathrm{e}}$ parties «Exemples tirés de la pratique», à paraître dans le Bulletin des médecins suisses.

17 Kuhn HP, Favre N. Bureau d'expertises de la FMH. Rapport annuel pour la période sous revue 2002. Bull Méd Suisses 2003;84(22):1160-3.
L'expert accepté et mandaté engage les mesures nécessaires pour éclaircir le cas et convoque le patient à un examen et à une entrevue. Il écoute ensuite la partie adverse en vertu du droit d'être entendu. Il utilise le schéma de questions pour les expertises extrajudiciaires de la FMH, qui met en évidence, sur plusieurs pages, les points importants d'un mandat habituel et tout particulièrement ceux qui concernent l'appréciation de la faute et la question de la causalité. D'éventuelles questions concrètes supplémentaires doivent être posées d'un commun accord par les parties. On y recourt dès lors le moins possible, afin d'éviter des débats interminables. Une question délicate est celle de l'information du patient qui ne relève pas, en tant que question de droit, du domaine central de l'expert médical, mais qui joue néanmoins un rôle en lien avec la question de l'examen et du traitement (indication) corrects sur le plan médical. Si l'indication de l'opération est discutable, l'expert cherche à savoir si le «principe de la démarche la plus sûre» a été suivi ou, autrement dit, si l'on a épuisé toutes les mesures d'investigation diagnostiques et thérapeutiques conservatoires, si l'on a soigneusement évalué les chances et les risques d'une approche plus invasive en intégrant le patient à la discussion [15]. L'expertise doit être livrée dans les trois mois, une règle en pratique difficile à tenir. L'expertise livrée, la tâche du bureau d'expertises est terminée. Les parties sont libres dans l'appréciation de l'expertise et, selon l'art. $15 \mathrm{du}$ règlement, elles peuvent demander un complément si la demande est fondée et que l'expertise se révèle lacunaire ou contradictoire. L'expérience montre que les compléments d'expertise n'améliorent que rarement l'élucidation du cas, l'expert ayant tendance à consolider son avis par d'autres arguments et éléments bibliographiques, au lieu de revoir d'un œil critique ses réflexions et conclusions à la lumière des remarques émises. Les expertises de la FMH livrent tout de même un fondement utile pour la suite du traitement du cas [16]. Le nombre des fautes reconnues a légèrement augmenté ces dernières années, se situant à $30,8 \%$ pour la période 1982-2002. Selon les constatations du Bureau, ce chiffre dépend de la qualité du réseau de conseil médical dont disposent les avocats et conseillers de patients. En 2002, 133 expertises ont été menées, dont 55 cas ou 41,4\% ont conclu à une faute de traitement [17]. A la connaissance de l'auteur, il n'existe pas en Suisse d'autre statistique étayée à ce sujet. Ici comme ailleurs, il faut s'attendre à l'existence de cas non recensés, dont le nombre ne doit pas être sous-estimé. 